\title{
Coding Synthetic Chemistry Strategies into Bacterial Designer Cells
}

Yu-Chang Liu, a Zhong-Liu Wu,b and Jan Deska a*

(a) Department of Chemistry, Aalto University, Espoo, Finland

(b) Key Laboratory of Environmental and Applied Microbiology, Chengdu Institute of Biology, Chinese Academy of Sciences, Chengdu, China

jan.deska@aalto.fi

\begin{abstract}
Following a synthetic chemistry blueprint for the valorization of lignocellulosic platform chemicals, this study showcases a so far unprecedented approach to implement non-natural enzyme modules in vivo. For the design of a novel functional whole cell tool, two purely abiotic transformations were incorporated into a recombinant bacterial host that allows production of complex lactone building blocks. This whole cell system streamlines the synthetic cascade, eliminates isolation and purification steps, and provides a high degree stereoselectivity that has so far been elusive in the chemical methodology.
\end{abstract}

The systematic expansion of the reaction scope of natural systems beyond the biosynthetically encoded transformations has received considerable attention over the past decade. Here, chemically relevant key transformations are used as a blueprint for the design of new-to-nature enzyme catalysts. The different approaches to reach abiotic functions can range from the rational de novo design of biocatalysts, ${ }^{[1]}$ over directed evolution to nurture trace activities, ${ }^{[2]}$ all the way to repurposing of wild-type proteins based on the exploitation of intrinsic promiscuity features ${ }^{[3]}$ or external triggers such as photoexcitation. ${ }^{[4]}$ While the reverse biomimetics approach, using chemistry as an inspirational source for the development of biological tools, can be seen as one of the current success stories in the field of biocatalysis, so far in vivo applications of artificial enzyme modules in genetically tailored microbes have been rare. ${ }^{[5]}$ More importantly, these examples were always limited to the expression of a single protein featuring the artificial function without its incorporation into an existing metabolic network that could make use of the new reactivity. a key chemical
transformations

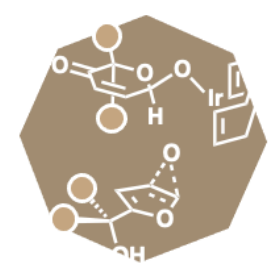

unprecedented in nature, yet template for the design of new enzymes

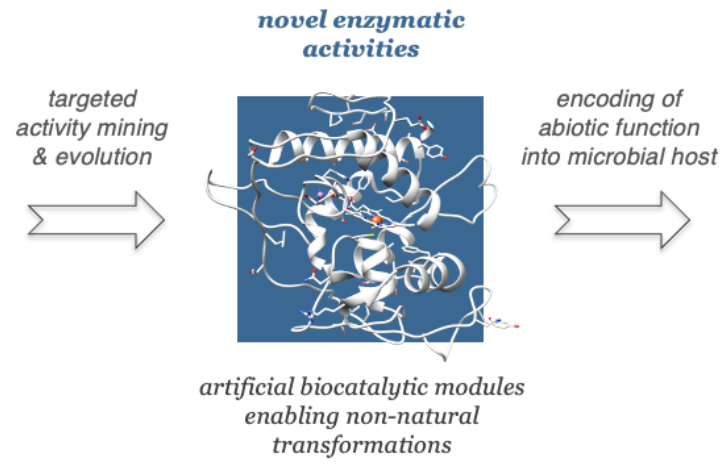

next generation metabolic pathway design

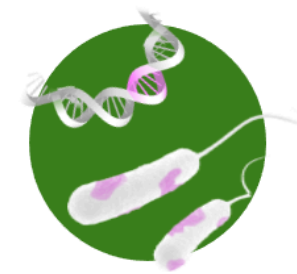

tailor-made cellular factories based on traditional chemical strategies

b

This study: a tailor-made cell factory for the valorization of lignocellulosic platform chemicals

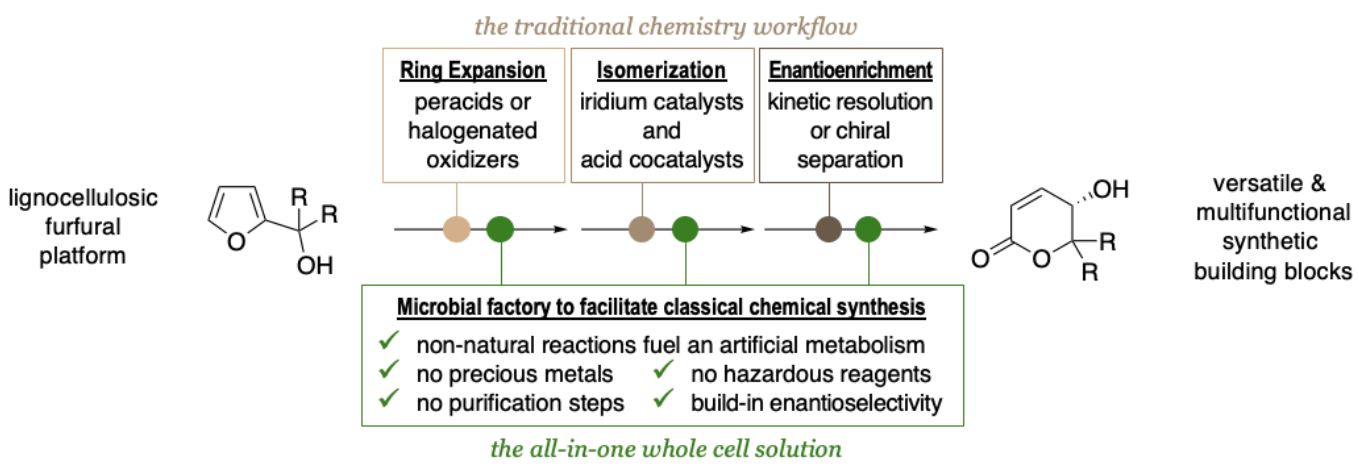

Figure 1 | Intended workflow from a traditional chemical synthesis strategy towards chemoinspired tailor-made cell factories: a inspiration from chemical methodology informs the development of novel biocatalysis modules which in turn allow to break away from the current limitations in metabolic engineering; $\mathbf{b}$, streamlining existing valorization strategies of bio-renewables through novel designer cells. 
Currently, there seems to be a missing link between these reaction-driven advances in biocatalysis and the obvious long-term application, that is, to make new-to-nature enzymology part of modern metabolic engineering. ${ }^{[6]}$ Offering very elegant and highly streamlined solutions for the biological synthesis of complex metabolites, the development of microbial producers of valuable chemicals such as pharmaceuticals ${ }^{[7]}$ or polymer precursors ${ }^{[8]}$ has recently seen a tremendous boom where transgenic strains combine elements of different biosynthetic pathways to assemble novel bioproduction platforms. Nevertheless, while these techniques become rapidly more elaborate and effective, the limited reaction portfolio of native biosynthesis hampers an even wider and more marketfocussed implementation. Encoding abiotic functions into microbial hosts not just as a stand-alone feature but as central element in a next-generation pathway design appears as promising approach that allows to produce valuable chemicals that would not necessarily be accessible by relying on anything found in the realm of normal biosynthesis (Figure 1a).

In this case study, we disclose a first successful implementation of multiple non-natural tools in a whole cell scenario. The furfural platform is widely considered as an important pillar of bio-renewable value chains. ${ }^{[9]}$ The furans as major intermediates in lignocellulosic biorefinery offer a rich reactivity for further derivatizations and synthetic chemistry has successfully developed routes for their valorization to versatile and multifunctional building blocks. ${ }^{[10]}$ However, the existing chemistry is not particularly green, relies on hazardous or non-sustainable reagents/catalysts and suffers selectivity issues. In this work, we translate the previously reported traditional chemistry workflow into a bacterial system that interconnects native and non-native reactivities to arrive at a strain that effectively mimics the chemical blueprint (Figure 1b). To the best of our knowledge, this constitutes the first example of an all-in-one whole cell solution where a microbial factory facilitates classical chemical synthesis in a multi-step fashion.

The key obstacle in the development of a whole cell solution, in which non-natural transformations ought to be utilized in reaction cascades outside the host organism's natural metabolism, is clearly the identification of proteinbased solution for the necessary abiotic functions. Not just in the case of our furfural refinement approach, previously identified biocatalytic tools can only act as a starting point for an activity mining as whole cell cascades demand much stricter abidance to a range of parameters and properties than in vitro enzyme catalysis. Combining two non-natural transformations, the implementation of our furfural platform valorization strategy required the identification of at least two individual enzymes with the prerequisites to a) show high activity in the desired Achmatowicz rearrangement and the borrowing hydrogen redox isomerization, respectively, b) operate in a nicotinamidedependent fashion in order to effectively couple the processes with each another, and c) warrant the compatibility of co-expression between multiple heterogenous genes in a single host cell, making sure each component can work in the designated pathway. Furfuryl alcohol 1a was chosen as model compound for the development of a whole cell furan refinement system. While traditional synthetic chemistry relies on brominating agents such as $\mathrm{N}$-bromosuccinimide for the ring expansion reaction (Table 1, entry 1), ${ }^{[11]}$ the combination of chloroperoxidase and glucose oxidase delivers an elegant biocatalytic alternative and provides comparable yields of the intermediate pyranone $\mathbf{2 a}$ (Table 1 , entry 2). ${ }^{[12]}$ As fungal enzyme with a rich glycosylation pattern and peroxide-dependence instead of the desired nicotinamidedependence, chloroperoxidase however does not comply well with a design based on a bacterial host organism, and hence, we investigated a small set of monooxygenases as more compatible alternatives. The introduction of the genes corresponding to cytochrome P450 (from B. megaterium), cytochrome P108N7 (from R. wratislavensis) and styrene monooxygenase (from Pseudomonas sp LQ26), respectively, into $E$. coli BL21(DE3) $\triangle$ nemA resulted in recombinant cells that were cultivated and analyzed for reactivity in the conversion of 1a (Table 1, entries 3-5). The $\Delta$ nemA strain was chosen to prevent undesired reductase activity on the enone-type intermediates/product. ${ }^{[13]}$ Only whole cell catalysts carrying the flavin-dependent styrene monooxygenase exhibited significant activity and to our delight, the desired pyranone 2 a could be obtained with a high selectivity of $73 \%$. Next, the second key transformation to the target lactone product $\mathbf{3 a}$ was addressed with a screening on potential redox isomerization biocatalysts. Originally described by Tang and Guo using an iridium catalyst and 2,4-dichlorobenzoic acid as co-activator (Table 1 , entry 6 ), ${ }^{[14]}$ in 2018 , we reported on the utilization of commercial alcohol dehydrogenases as proteinogenic counterpart for the borrowing hydrogen transformation of 2a to yield 3a not just in superior yields, but with very high enantioselectivity of up to $96 \%$ ee (Table 1, entry 7), a feature that has so far not been achieved in this reaction by transition metal-based catalysts. ${ }^{[15]}$ The same E. coli BL21(DE3) $\Delta$ nemA was exploited as host for the expression of five different nicotinamide-dependent dehydrogenases (Table 1, entries 8-12). Whereas the dehydrogenation to the corresponding ketolactone $4 \mathrm{a}$ was observed as a more or less common feature, only the dehydrogenase from Lactobacillus kefir was able to perform the full redox isomerization (lactol dehydrogenation and ketoreduction). Here, LkADHexpressing E. coli did not only convert rac-2a quantitatively (at up to $10 \mathrm{~g} / \mathrm{L}$ within $4 \mathrm{~h}$ ), but in an enantioconvergent manner rendered the product lactone $\mathbf{3 a}$ as single enantiomer ( $e e>99 \%$ ), exceeding the selectivities of any commercially available biocatalyst so far. However, to our very surprise, four out of five incubations of $\mathbf{2 a}$ with the recombinant cells actually delivered significant amounts of furfuryl alcohol 1a, reaching up to $30 \%$ in case of Chryseobacterium ADH (Table 1, entry 11). 


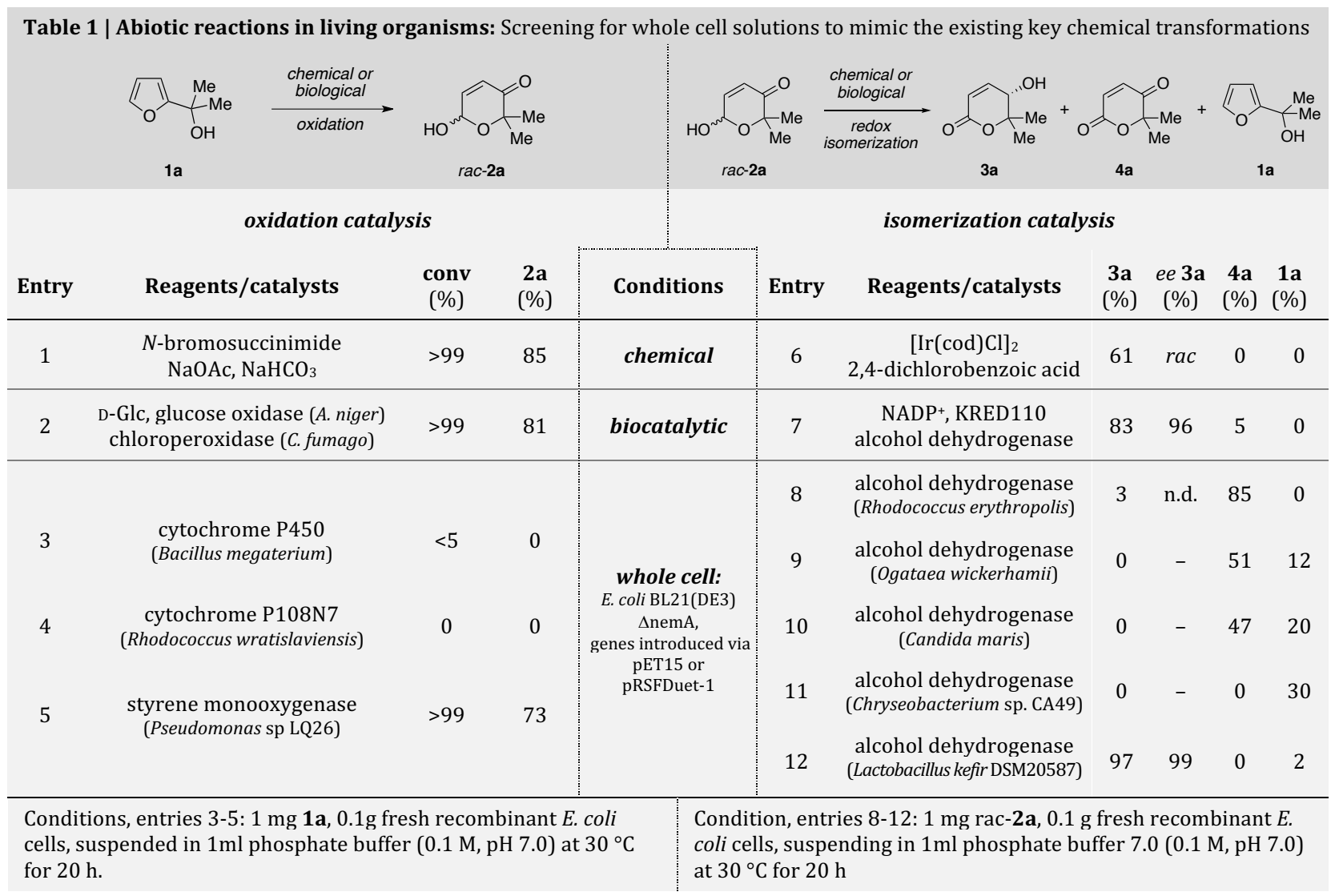

While the original screening to identify suitable expressible enzyme modules for the two key transformations was successful, the rather general appearance of the furfuryl alcohol 1a, the actual starting point of a potential synthetic cascade, with most of the tested dehydrogenases, raised questions on the validity of our design. In order to assess the risk of a non-productive loop, perpetually converting $\mathbf{1 a}$ to $\mathbf{2 a}$ and straight back to 1a under the consumption of reduction equivalents, the most effective isomerase module based on L. kefir alcohol dehydrogenase was exposed to varying levels of reductive pressure (Scheme 2). Resting cells of the bacterial host overexpressing only the artificial isomerase in a nonreductive medium clearly performed their designated role with virtue, yielding the optically pure lactone $(S)-3 \mathbf{a}$ in $97 \%$, with only traces of the furfuryl alcohol $\mathbf{1 a}$. Supplementing the medium with glucose in order to drive the endogenous production of reducing nicotinamides, furan production was slightly upregulated, yet the desired lactone remained the major metabolite. In stark contrast, introducing the glucose dehydrogenase gene alongside the artificial isomerase in a co-expression system, the modified recombinant bacterial host also converted the pyranone $\mathbf{2 a}$ with ease, however, the selectivity switched entirely in favor of the furan product. This conversion of $\mathbf{2}$ to $\mathbf{1}$ represents a so far unprecedented direct retroAchmatowicz reaction, that without doubt merits further attention as a possible biocatalytic tool. In the development of an effective whole cell cascade to produce enantiomerically pure lactones, however, this parasitic
retro-Achmatowicz ring contraction poses a significant challenge demanding strict regulation of the redox balance within the cellular factory.
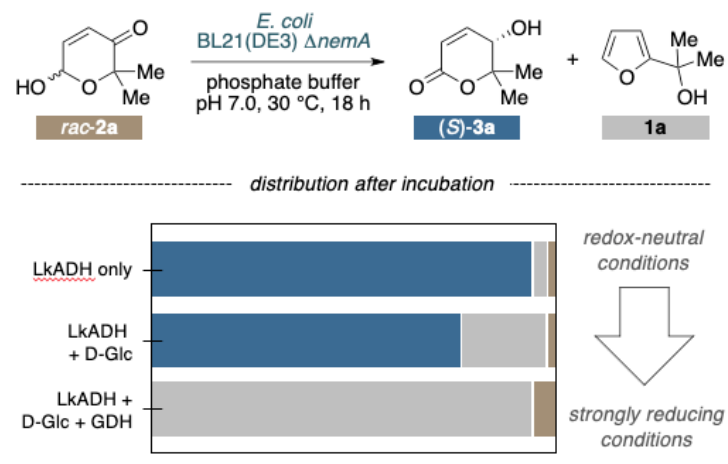

Figure 2 | Unprecedented retro-Achmatowicz pathway as side reaction under reductive pressure.

The initial investigations in the desired route to produce enantiopure lactones directly from biorefinery furans revealed an extended picture of fundamental steps and dependencies (Scheme 3), and a number of possible points of intervention to regulate the biosynthetic machinery towards the chiral heterocyclic products. In an initial ring expansion, a monooxygenase converts the biogenic furans $\mathbf{1}$ to the intermediary pyranones $\mathbf{2}$ with consumption of dioxygen and reduced nicotinamides. From here, a catalytic bifurcation emerges, where the productive or non- 
productive outcome is decided by the selectivity to either reductively or oxidatively activate 2 . The desired route (isomerase-mode) proceeds with the oxidation of the hemiacetal moiety through an alcohol dehydrogenase utilizing $\mathrm{NAD}(\mathrm{P})^{+}$to form the ketolactones 4 alongside with NAD(P)H. With the same - or a second, complementary dehydrogenase the ketone is finally reduced to give rise to the hydroxylactones 3 and recycle $\mathrm{NAD}(\mathrm{P})^{+}$to drive the redox-self-sufficient isomerization. If oxidation of $\mathbf{2}$ remains slow, the risk for a reductive parasitic off-cycle pathway is increased in which the alcohol dehydrogenase consumes $\mathrm{NAD}(\mathrm{P}) \mathrm{H}$ to deliver the reduced six-membered hydroxylactol 5 . After ring contraction to the corresponding five-membered lactol 6, spontaneous dehydration closes the non-productive cycle and delivers the furanoic starting material, after net consumption of two equivalents of reducing nicotinamide. Both the selectivity of the alcohol dehydrogenase(s) as well as the balance between oxidizing and reducing cofactors in the bacterial designer cells will thus influence this delicate network of reactions. Especially the ambiguity regarding reducing cofactors, being crucial to catalyze the furan oxygenation while also offering an entry into the undesired off-cycle need to be carefully addressed in the actual cascade design.

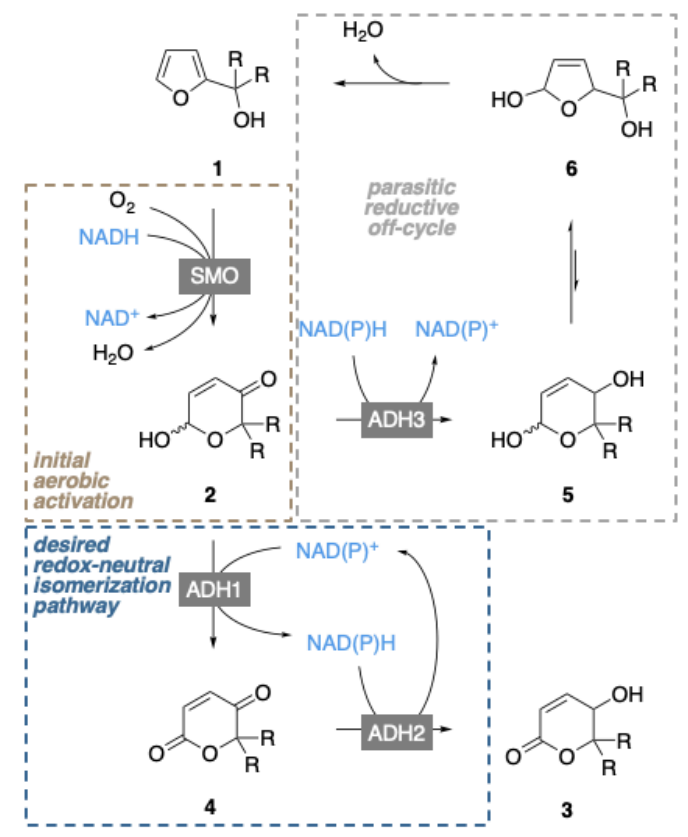

Figure 3 | Productive and non-productive pathways for the biotransformation of furfuryl alcohols based on a cascade design that translates the chemistry blueprint literally.

In a first attempt to assemble a suitable cellular factory, the coding sequences of styrene monooxygenase and LkADH were amplified and engineered into the commercially available pRSFDuet-1 $\mathrm{1}^{\mathrm{T}}$ vector, giving two coexpression plasmids SMO-LK and LK-SMO under the control of two T7 promoters. The two plasmids were transformed into E.coli $\Delta$ nemA to produce E.coli (SMO-LK) and E.coli (LK-SMO), respectively. The strains were cultivated in LB medium with kanamycin, the target proteins were successfully expressed, and the fresh resting cells were employed to transform model substrate 1a. Interestingly, the two different architectures exhibited an orthogonal selectivity profile. Whereas E. coli (LK-SMO) only performed the initial furan oxidation with no traces of the desired lactone (Table 2, entry 2), E. coli (SMO-LK) managed to act as bacterial producer for the final product (S)-3a with an optical purity of $99 \%$ (Table 2 , entry 3 ). The excessive amount of remaining furan substrate left in either of the reaction mixtures, however, is indicative of an inefficient supply of reduced nicotinamide, and perhaps also a result of issues related to the co-expression of Lk$\mathrm{ADH}$ and SMO from the same plasmid. Considering the highly oxidative capability of SMO on 1a as described above, we decided to ameliorate nicotinamide regeneration in order to support the SMO-catalysed oxidation by means of another dehydrogenase in the single whole cell system. SMO is a strictly NADH-dependent monooxygenase whilst Lk-ADH is preferentially using NADPH, allowing to selectively address only one of the key oxidoreductases. The coding sequence of formate dehydrogenase (FDH) was engineered into the $\mathrm{pET}-15 \mathrm{~b}$ vector, and transformed into E. coli BL21(DE3) $\triangle$ nemA together with the pRSFD-SMO-LK plasmid yielding E. coli (SMO-LK/FDH). Unfortunately, this construct proved to be ineffective as no conversion of furan 1a was detected even after an extended incubation period (Table 2, entry 4).

The previously elaborated reaction network, however, also offers an alternative way to supply reduced nicotinamides for the SMO-mediated Achmatowicz oxygenation. As discovered in the initial screening, certain alcohol dehydrogenases do indeed engage in the dehydrogenation of $\mathbf{2}$ and provide ketolactone $\mathbf{4}$, although they are incapable to conduct the complete redoxisomerization. Introduction of one of those biocatalytic tools would not only strongly influence the catalytic bifurcation in favor of the desired oxidative activation, but it would also create a redox-self-sufficient unit to push the so far ineffective furan rearrangement by improved supply of NADH fueling the SMO module. Therefore, alcohol dehydrogenase ReADH with its NADH cofactor preference (and also its low ketoreductase activity in the reduction of $\mathbf{2}$ or $\mathbf{4}$ ) was chosen a supplementary module alongside SMO and LkADH in vivo. To test our hypothesis, a binary co-expression system combining ReADH and SMO was assembled and in fact delivered almost exclusively the ketolactone $\mathbf{4 a}$ (Table 2, entry 5). In order to optimize the expression level of the three genes required for the complete cascade, the coding sequences of SMO, LKADH and READH were engineered on pRSFDuet- $1^{\mathrm{T}}$ and $\mathrm{pET}-15 \mathrm{~b}$ to give a total of four different expression cassettes, namely, SLR1 (pRFDuet-SMO-LK/pET15b-RE), SLR2 (pRFDuet-LK-SMO/pET15b-RE), SLR3 (pRFDuet-RELK/pET15b-SMO), SLR4 (pRFDuet-Re-SMO/pET15b-LK). Each operon was under the control of individual T7 promotors. The four sets of plasmids were transformed into E.coli BL21(DE3) $\triangle$ nemA, respectively, and the whole cell catalysts were prepared as described before. The four strains were applied to the biotransformation of $\mathbf{1 a}$ and 


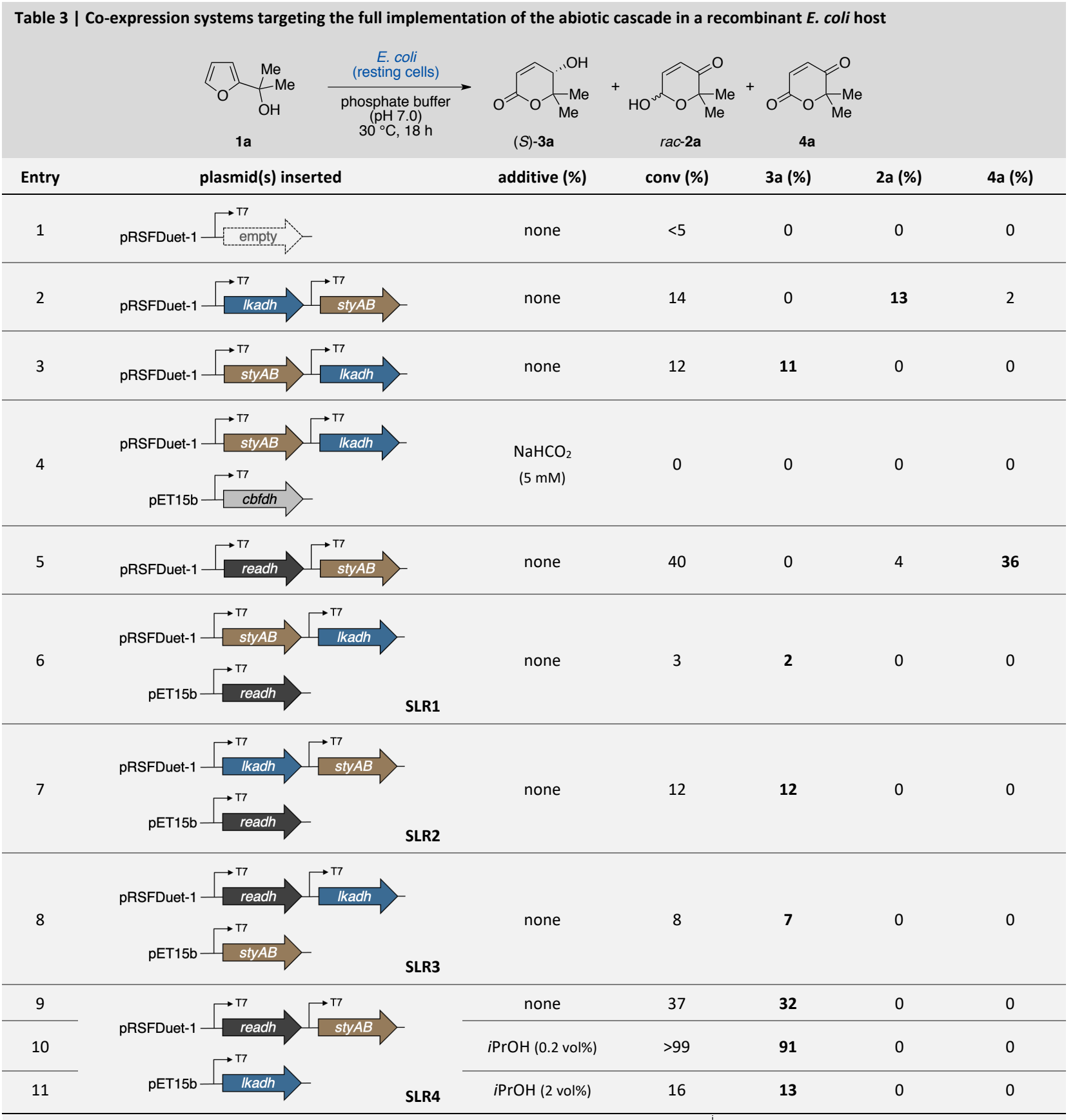

while all exhibited some activity to produce the lactone $(S)$ 3a (Table 2, entries 6-9), SLR4 showed the best performance without the accumulation of any intermediates. Even though the first two steps in the design include SMO and ReADH as redox-self-sufficient biocatalytic entity, ${ }^{[16]}$ the overall process requires external reductants. Thus, utilization of the commonly employed cofactor recycling using isopropanol to drive dehydrogenase processes appeared as a logical measure to overcome the mediocre yields of $\mathbf{3 a}$ (max. 32\%). Gratifyingly, supplementing the medium with $0.2 \%(\mathrm{v} / \mathrm{v})$ isopropanol resulted in a highly effective cellular factory providing not only full conversion but $91 \%$ selectivity for the enantiopure lactone (S)-3a. (Table 2, entry 10). Excessive supply of isopropanol on the other hand led to an apparent inhibition, most likely by deviating the artificial metabolism towards the non-productive off-cycle (Table 2, entry 11).

Taking into account the apparent side reaction profile and the individual characteristics of the three enzyme modules, the most likely cascade network was concluded to follow the sequence depicted in Figure 4a. In contrast to the original design (Figure 2), our most effective cellular factory deviates from the initial draft in that the redox-selfsufficient isomerase module (i.e. net-neutral in NAD(P)H with one single dehydrogenase biocatalyst) has been replaced by a net-NADH-neutral conversion of the furfuryl alcohols (1) to the ketolactone intermediates (4) by cooperative action of styrene monooxygenase and ReADH, while the net-reducing event has been relocated towards 
a

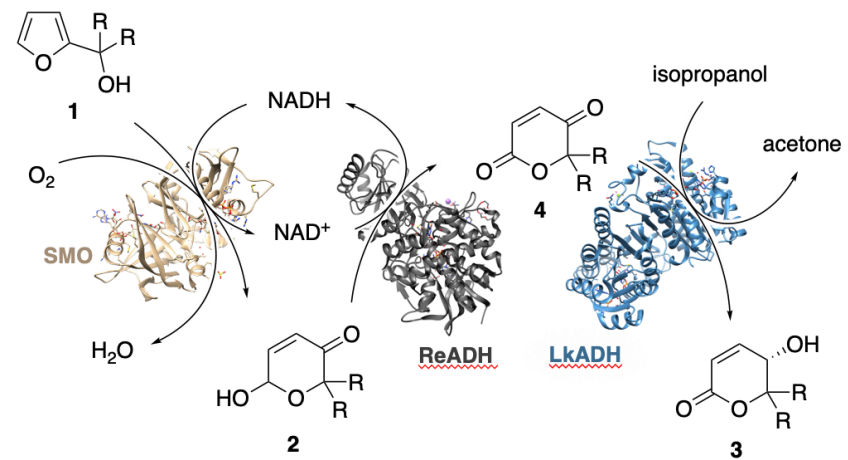

b

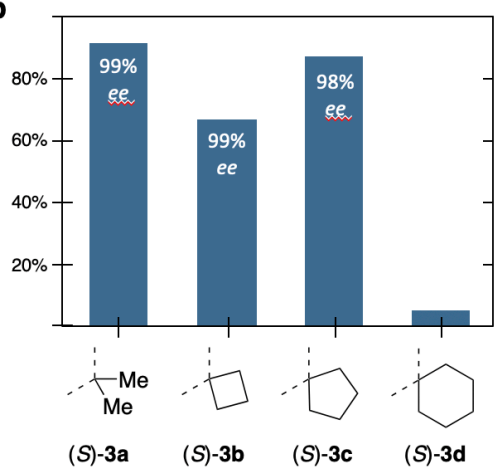

Figure 5 | Production of enantiopure hydroxylactones in a tailor-made E. coli strain: a, biocatalytic network enabling a direct conversion of furfuryl alcohols. $\mathbf{b}$, substrate scope of preparative scale whole cell biotransformations.

the end of the sequence. With this ternary expression system in place, a powerful whole cell assembly for the direct production of enantiomerically pure delta-lactones from basic wood-derived furan precursors was obtained. To our delight, the bacterial construct was not limited to the model furan 1a but accepted also structural variations (Figure 4b). Incubation with 1-furylcycloalkanols resulted in the production of the synthetically highly interesting spirolactones (S)-3b and (S)-3c in good yields and excellent optical purities of greater $98 \%$. The current limitation of this initial cellular factory design appears to lie in the substrate lipophilicity and or sterics as cyclohexanol 1d was only poorly accepted by the same microbial system. Future investigations on the herein presented design will therefore target the influence of cosolvents and emulsifiers as well as controlling fluxes that may help to expand the substrate scope in order to make full use of this exciting new biological tool for the valorization of simple lignocellulosic platform chemicals to stereochemically defined building blocks for fine chemicals, pharmaceuticals and fragrances.

In summary, we were able to successfully incorporate a truly non-natural reaction cascade into a simple bacterial host, and to fine tune the co-expression system by rather simple means to act as highly selective producer of multifunctional synthetic building blocks from a basic lignocellulose-derived feed. The thus obtained bioproduction platform exhibits a range of benefits over its organic-synthetic template, as the valorization of biorefinery furan in the designer cells proceeds without any intermediate isolation or purification steps, rendering analytically pure lactones after a simple extraction in very high overall yields. Avoiding any kind of hazardous or precious metal-based additives, the biological interpretation of this synthetic chemistry strategy appears not just much more sustainable than the traditional blueprint but also benefits from the intrinsic nature of the key enzyme functions in that the products are generally obtained with excellent enantioselectivity. In addition, the same study provided also whole new insights into the established underlying chemistry of the target process, where the discovery of a so far unprecedented retroAchmatowicz reaction will unequivocally serve as starting point for mechanistic investigations on this transformation.

\section{Acknowledgements}

We gratefully acknowledge financial support of this study by the Academy of Finland (298250 (JD) \& 324854 (YCL)) and the Novo Nordisk Fonden (NNF170C0025092 (YCL)).

\section{Keywords}

artificial enzyme modules, bio-renewables, platform chemicals, whole cell biotransformations, chemoinspired

\section{References}

[1] (a) J. B. Siegel, A. Zhanghellini, H. M. Lovick, G. Kiss, A. R. Lambert, J. L. St. Clair, J. L. Gallaher, D. Hilvert, M. H. Gelb, B. L. Stoddard, K. N. Houk, F. E. Michael, D. Baker, Science 2010, 329, 309-313; (b) R. Blomberg, H. Kries, D. M. Pinkas, P. R. E. Mittl, M. G. Grütter, H. K. Privett, S. L. Mayo, D. Hilvert, Nature 2013, 503, 418-421.

[2] (a) P. S. Coelho, E. M. Brustad, A. Kannan, F. H. Arnold, Science 2013, 339, 307-310; (b) V. Tyagi, G. Sreenilayam, P. Bajaj, A. Tinoco, R. Fasan, Angew. Chem. Int. Ed. 2016, 55, 1356213566; (c) C. K. Prier, R. K. Zhang, A. R. Buller, S. BrinkmannChen, F. H. Arnold, Nature Chem. 2017, 9, 629-634; (d) R. K. Zhang, K. Chen, X. Huang, L. Wohlschläger, H. Renata, F. H. Arnold, Nature 2019, 565, 67-72; (e) N. W. Goldberg, A. M. Knight, R. K. Zhang, F. H. Arnold, J. Am. Chem. Soc. 2019, 141, 19585-19588;

[3] (a) C. Jäger, J. Deska, ChemRxiv 2021, 10.26434/ chemrxiv.14562048, (b) D. Thiel, F. Blume, C. Jäger, J. Deska, Eur. J. Org. Chem. 2018, 20, 2717-2725; (c) J. Naapuri, J. D. Rolfes, J. Keil, C. Manzuna Sapu, J. Deska, Green Chem. 2017, $19,447-452$.

[4] L. Schmermund, V. Jurkaš, F. F. Özgen, G. D. Barone, H. C. Büchsenschütz, C. K. Winkler, S. Schmidt, R. Kourist, W. Kroutil, ACS Catal. 2019, 9, 4115-4144.

[5] (a) P. S. Coelho, Z. J. Wang, M. E. Ener, S. A. Baril, A. Kannan, F. H. Arnold, E. M. Brustad, Nature Chem. Biol. 2013, 9, 485-487; (b) S. B. J. Kan, R. D. Lewis, K. Chen, F. H. Arnold, Science 2016, 354, 1048-1051.

[6] (a) S. P. France, L. J. Hepworth, N. Turner, S. L. Flitsch, ACS Catal. 2017, 7, 710-724; (b) K. B. Otte, B. Hauer, Curr. Opin. Biotechnol. 2015, 35, 16-22.

[7] (a) D.-K. Ro, E. M. Paradise, M. Ouellet, K. J. Fisher, K. L. Newman, J. M. Ndungu, K. A. Ho, R. A. Eachus, T. S. Ham, J. Kirby, M. C. Y. Chang, S. T. Withers, Y. Shiba, R. Sarpong, J. D. Keasling, Nature 2006, 440, 940-943; (b) X. Luo, M. A. Reiter, L. d'Espaux, J. Wong, C. M. Denby, A. Lechner, Y. Zhang, A. T. Grzybowski, S. Harth, W. Lin, H. Lee, C. Yu, J. Shin, K. Deng, V. 
T. Benites, G. Wang, E. E. K. Baidoo, Y. Chen, I. Dev, C. J. Petzold J. D. Keasling, Nature 2019, 567, 123-126.

[8] N. Oberleitner, A. K. Ressmann, K. Bica, P. Gärtner, M. W. Fraaje, U. W. Bornscheuer, F. Rudroff, M. D. Mihovilovic, Green Chem. 2017, 19, 267-271.

[9] R. Mariscal, P. Maireles-Torres, M. Ojeda, I. Sádaba, M. López Granados, Energy Environ. Sci. 2016, 9, 1144-1189.

[10] (a) A. K. Ghosh, M. Brindisi, RSC Adv. 2016, 6, 111564111598; (b) C. Verrier, S. Moebs-Sanchez, Y. Queneau, F. Popowycz, Org. Biomol. Chem. 2018, 16, 676-687.
[11] J. Deska, D. Thiel, E. Gianolio, Synthesis 2015, 47, 3435-3450.

[12] D. Thiel, D. Doknić, J. Deska, Nat. Commun. 2014, 5, 5278;

[13] K. Miura, Y. Tomioka, H. Suzuki, M. Yonezawa, T. Hishinuma, M. Mizugaki, Biol. Pharmaceut. Bull. 1997, 20, 110-112.

[14] H.-Y. Wang, K. Yang, S. R. Bennett, S.-R. Guo, W. Tang, Angew. Chem. Int. Ed. 2015, 54, 8756-8759.

[15] Y.-C. Liu, C. Merten, J. Deska, Angew. Chem. Int. Ed. 2018, 57, 12151-12156.

[16] Y.-C. Liu, Z.-L. Wu, Chem. Commun. 2016, 52, 1158-1161. 